\title{
Iris Recognition using Left and Right Iris Feature of the Human Eye for Bio-Metric Security System
}

\author{
B. Thiyaneswaran \\ Assistant Professor, ECE, \\ Sona College of Technology \\ Salem, Tamilnadu, India.
}

\author{
S. Padma \\ Professor, EEE \\ Sona College of Technology \\ Salem, Tamilnadu, India.
}

\begin{abstract}
Iris recognition plays an important role in the Biometric authentication. The eye lids, lashes and flash light impressions are hazard, which in turn reduces successive iris recognition rate. The proposed method includes the preprocessing of images such as image filter, morphological operations, and edge detection, which finds the exact pupil part. The proposed method uses the MLRP algorithm, to identify the exact iris layers rather than the existing methods. The key feature is extracted in iris layer. The method is applied on both left and right iris, which gives unique key between left and right eye for every person. The extracted key feature identifies the eye even in the different eye position, which gives the repeatability. The proposed method is tested with the CASIA data base iris images, which consists of left and right eye set for the different human. The proposed method reduces the FAR to $15.6 \%$ and FRR to $14 \%$.
\end{abstract}

\section{General Terms}

Biometric - Image processing, Image repeatability.

\section{Keywords}

Unique key, Median filter, canny edge detection, flash noise, FAR, FRR.

\section{INTRODUCTION}

The biometric systems includes the human parts such as finger print, palm print, hand geometry, face, ear, iris, Retina, signature, DNA, key stroke, voice recognition, body odour, etc., [1]. The eye based biometric system plays an important role. In the eye bases system, the retina and iris are the important sources for the biometrics.

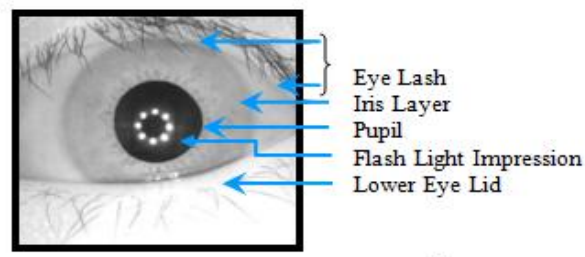

Fig 1: Structure of Human Eye

The eye biometrics provides the highly reliable in the human authentication. The retina photography makes a complex process, as compared with the iris [2]. During eye transportation if the eye is removed from human, the iris structure gets changed. Ageing also not affects the iris structure. The iris layer dimension provides the uniqueness feature [3] [4]. The iris dimensions are differs for left and right eye, even for twins. The human eye consists of eye lids, eye lashes, iris, pupil, flash light impression on the pupil area as shown in the Figure-1. The eyelids, eye lashes and flash light impressions are the iris hazards [5]. The complete block diagram of proposed system is shown in the Figure 2. It consists of pre-processing stage, MLRP algorithm and image matching section.

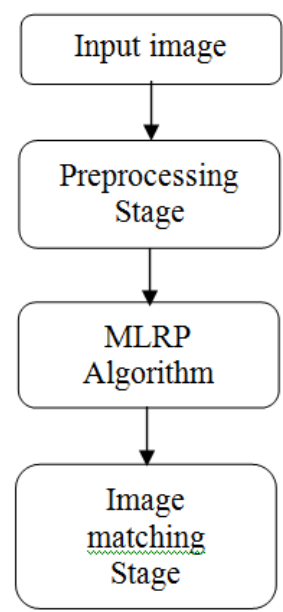

Fig 2: Block Diagram of Proposed system

\section{PREPROCESSING}

The image taken out is subject to the pre-processing stage. The image obtained was in the higher contrast and brightness. The direct process of the image leads to more amount of unwanted eye information. The iris and the pupil area are to be separated from the other eye data. The Block Diagram of preprocessing stage is shown in the Figure 3. The median filter is preferred over the other filters such as wiener, kalman, orad, etc... The filter with suitable iteration is used to filter the image [13]. The Filtered image is subjected to Binary conversion process. The next objective is to find the pupil area. The pupil area is dark color than the other part of the eye. The binary conversion is depends on the thresholding value [8]. The thresholding is given as following:

$$
\begin{aligned}
& f(x, y)=1, \text { if } \mathrm{I}(\mathrm{x}, \mathrm{y}) \leq \mathrm{T} \\
& \mathrm{f}(\mathrm{x}, \mathrm{y})=0, \text { if } \mathrm{I}(\mathrm{x}, \mathrm{y}) \geq \mathrm{T}
\end{aligned}
$$




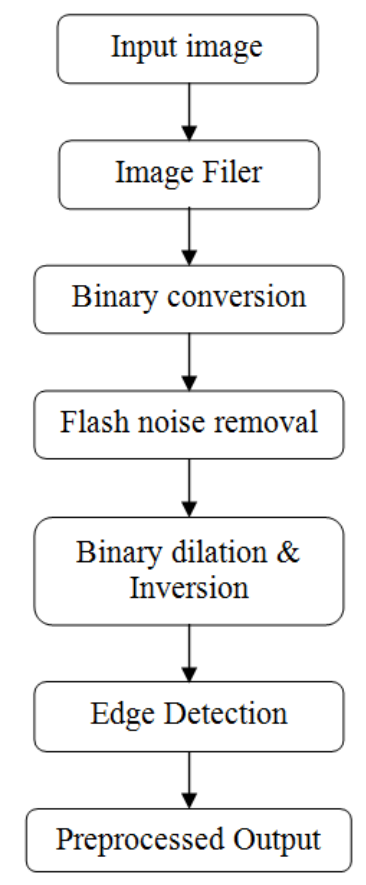

Fig 3: Block Diagram of Preprocessing Stage

If Let ' $\mathrm{T}$ ' be the threshold level, $l(x, y)$ is the actual threshold of the filtered image. After the threshold level comparison, the Pixel values are changed to level ' 0 ' and level ' 1 '. Flash light impression appears with the logic value ' 1 '. The morphological operator is applied to fill the logic ' 0 ' on the closed paths. In this the slender noises are appeared like smaller objects, which are completely filled with the logic ' 0 '. Dilation is a morphological process, in which the binary image is expanded from its original image. The way the binary image is expanded is determined by the structuring element [9]. The dilation process is similar to the convolution process. The slender noises are in the irregular shape, where as the pupil is in circular or ellipse shape. The light image structures are suppressed and the pupil image borders are completely connected [10] [16].

The binary inversion is the process of converting threshold values. The logic ' 0 ' pixels are converted to logic ' 1 ' and logic ' 1 ' pixels are converted to logic ' 0 '. Now the image was completely pre-processed. The exact pupil boundary is formed [12].

The purpose of edge detection is to detect strong and weak edges in iris region. In the experimental the canny edge detection technique gives out efficient edges for iris image, as compared with the other techniques [10] [14]. In Canny edge detection before trying to locate any edge; the first step is to filter out any noise in the original image. For filtering the noise the, Gaussian filter with $7^{\text {th }}$ iteration was selected by experimentally and noise was eliminated [6] [15].

\section{MLRP ALGORITHM}

The MLRP algorithm is used to identify the iris edges in accurate manner [2]. In the Figure 4, the inner circle shows the pupil and outer circle shows the iris circle. After the edge detection process, logic ' 0 'pixel value exits between the pupil layer and the iris layer. Then the following steps are to be followed for finding the exact iris layer.

\section{Step-1:}

The horizontal left and right pixel points are to be located in the pupil boundary. The pixels are exactly on the $0^{\circ}$ and $180^{\circ}$ of the pupil.

Step-2:

The horizontal left movement is followed from $0^{0}$ location of pupil. The movement starts at the pixel value of logic ' 1 '.

Step-3:

The straight line left side movement is followed till the pixel value ' 1 '. When it finds the logic value ' 1 ', that particular location is known as horizontal $0^{0}$ pixel location of the iris layer.

\section{Step-4:}

The horizontal right movement is followed from $180^{\circ}$ location of pupil. The movement starts at the pixel value of logic ' 1 '.

Step-5:

The straight line right side movement is followed till the pixel value ' 1 '. When it finds the logic value ' 1 ', that particular location is known as horizontal $180^{\circ}$ pixel location of the iris layer.

Step-6:

The multiple points are to be located from the $0^{\circ}$ and the $180^{\circ}$ pixels.

Step-7:

The $0^{0}$ and the $180^{\circ}$ pixels are in the logic value ' 1 '. From the $0^{0}$ the nearby logic ' 1 ' value is searched, it finds the next pixel location of the iris layer.

Step-8:

The step- 6 procedure is followed to find the more visible iris location, between $0^{0}$ to $\pm 5^{0}$ and similarly more visible points are located $180^{\circ}$ region. As the result the multiple points are located over the iris region as shown in the Figure 4.

Step-9:

The curve fitting algorithm is applied on the multiple points, which forms the exact iris layer over the iris hazards.

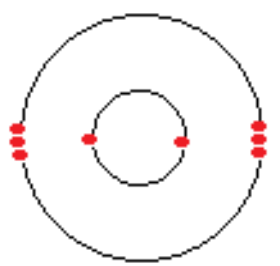

Fig 4: Proposed MLRP algorithm

\subsection{Feature Extraction}

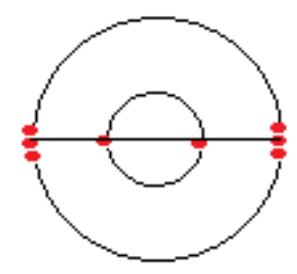

Fig 5: Hamming distance measurement 
The hamming distance between the horizontal points are to be calculated. The horizontal distance between the $0^{\circ}$ and the $180^{\circ}$ pixel locations are evaluated. In the Figure 5, horizontal line represents the distance between the $0^{\circ}$ and the $180^{\circ}$ pixels. The length of the line gives out the hamming distance between the diameters points [7]. This hamming distance is taken as unique key feature identification. The hamming distance is calculated for both the left and right iris of the human.

\section{IRIS MATCHING}

The image is stored in the data base. In this proposed method, the recognizable image is applied to the two pass comparison stage [11] [17]. The Figures 6 shows the flow of iris matching. Initially the right iris feature is compared; if the image is detected in the first pass comparison then its moved second pass. If the image is matched in the $2^{\text {nd }}$ pass, the result is positive [18]. The two pass stage considerably reduces the FRR and FAR.

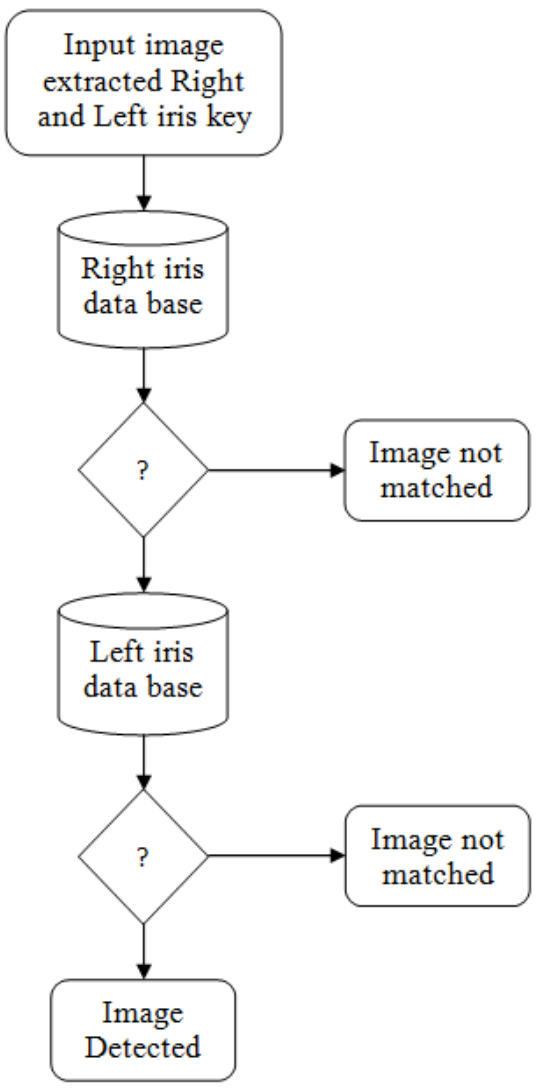

Fig 6: Iris matching flow

\section{RESULT AND DISCUSSION}

To evaluate the performance of the proposed system, extensive experiments were performed. Experiments were carried out on the iris database from the Chinese Academy of Science, Institute of Automation (CASIA). The data base includes left and right eye of the human. The proposed method is applied on both the left and right eye of an every human. The experiments were performed by using Matlab.

The input image is applied to the median filter, which blurs the image and the more amounts of unwanted information were removed shown in the Figures 8 . The filtered image is applied to the binary conversion process, which gives the pupil as the major object and some of the noise in the edges of the eye as shown in the Figures 9. The pupil also consists of flash noise. The morphological operation was performed, which fills the logic ' 0 ' value on the closed boundary. It removes the flash light impression and the slender noise components as shown in the Figure 11. Now the binary inverter operation was applied on the existing result. The pupil area was in logic ' 1 ' and other parts were in the logic' 0 ' as shown in the Figure 12. Now the pupil parameters such as centre and the area were calculated. The Figure 13 gives the pupil boundary over the input image.

The canny edge detection with suitable iteration was applied on the input image (shown in Figure 7), which detects the efficient visible iris layer as shown in Figure 14. It also shows that, there was a clear logic ' 0 ' pixel between the pupil layer and the iris layer. The MLRP algorithm was applied over the iris layer edged detected image. This in turn finds the more points over the iris layer as shown in the Figure 15. The curve fitting algorithm was applied and it finds the exact iris layer as shown in the Figure 16.

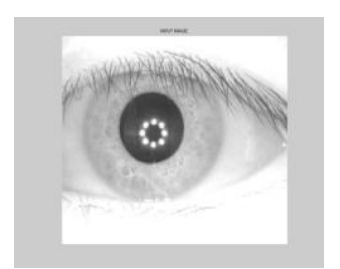

Fig.7 Input Image

Fig.9 Binary image

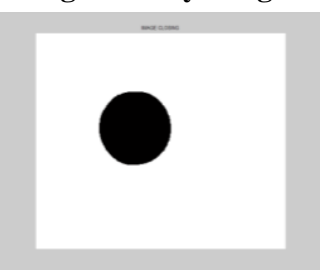

Fig.11 Dilated eye image

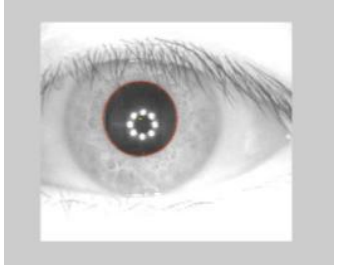

Fig.13 Pupil Boundary

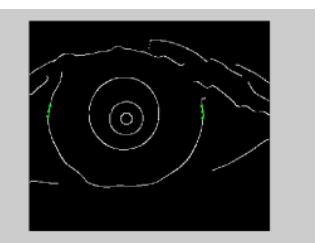

Fig.15MLRP Algorithm

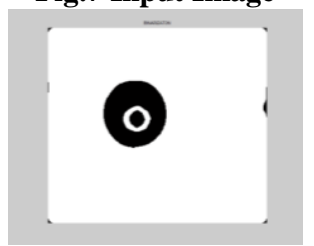

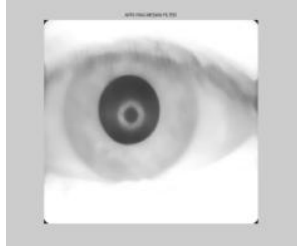

Fig.8 Filtered Image

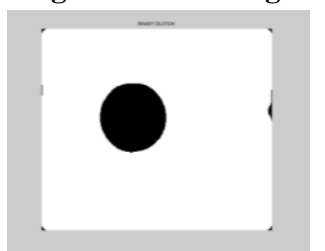

Fig.10 Flash noise removal

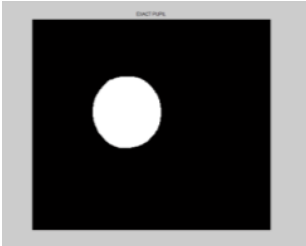

Fig.12 Binary inverted eye

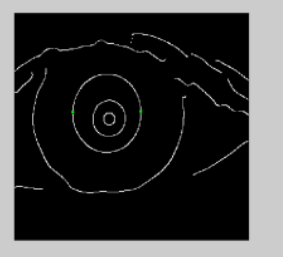

Fig.14 Canny edge detection

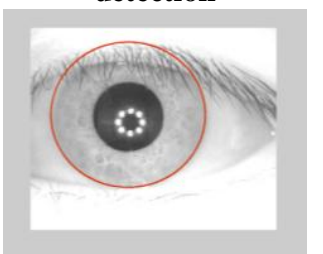

Fig.16 Iris Boundary 
The proposed algorithm was tested with the repeatability test process. The different eye positions of the single person were applied on the proposed algorithm. The algorithm also fits to recognize the iris image, even when it's taken out in the different angular position as shown in the Figure 17.

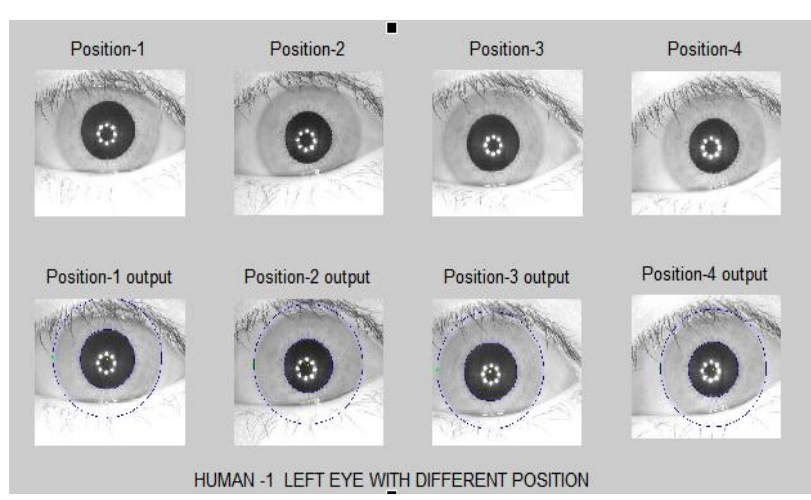

Fig 17: A human Eye with different angular position The Table 1 shows the sample of tested left and right eye of the individual humans. It also shows that the left and right hamming distance for a person gets varied and shows its uniqueness.

Table 1. Tested Iris Samples

\begin{tabular}{|c|c|c|c|c|}
\hline Person & Right eye & Left eye & $\begin{array}{c}\text { Hamming } \\
\text { distance } \\
\text { of right } \\
\text { iris }\end{array}$ & $\begin{array}{c}\text { Hamming } \\
\text { distance } \\
\text { of left } \\
\text { iris }\end{array}$ \\
\hline $\begin{array}{c}\text { Human } \\
1\end{array}$ & 0 & 0 & 215.12088 & 228.07578 \\
\hline $\begin{array}{c}\text { Human } \\
2\end{array}$ & 0 & 0 & 216.32910 & 217.83316 \\
\hline $\begin{array}{c}\text { Human } \\
3\end{array}$ & 0 & 0 & 207.71300 & 212.56880 \\
\hline $\begin{array}{c}\text { Human } \\
4\end{array}$ & 0 & 0 & 217.17181 & 222.17537 \\
\hline $\begin{array}{c}\text { Human } \\
5\end{array}$ & 0 & 0 & & \\
\hline
\end{tabular}

The corresponding plot of Table 1 is shown in the Figure 18. For example in the Table 1, we may take the human 4 key features. It clearly represents there is an uniqueness exists between left and right eye The difference was clearly shown illustrated in the Figure 18.

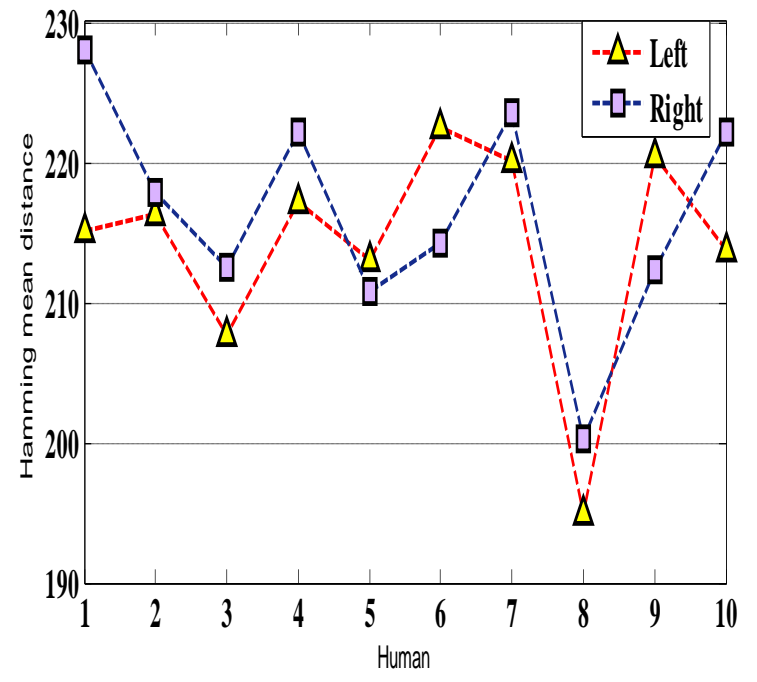

Fig 18: Iris uniqueness

The Table 2 shows the error rate comparison. In the existing method the FRR is about $20 \%$ and FAR is about $18 \%$. In the proposed method the error rates are reduced as FRR $=14 \%$ and FAR $=15.6 \%$. The corresponding bar representation is shown in Figure 19.

Table 2. False rejection and acceptance result

\begin{tabular}{|c|c|c|}
\hline Methodology & FRR & FAR \\
\hline Existing Fuzzy method & $20 \%$ & $18 \%$ \\
\hline Proposed Method & $14 \%$ & $15.6 \%$ \\
\hline
\end{tabular}

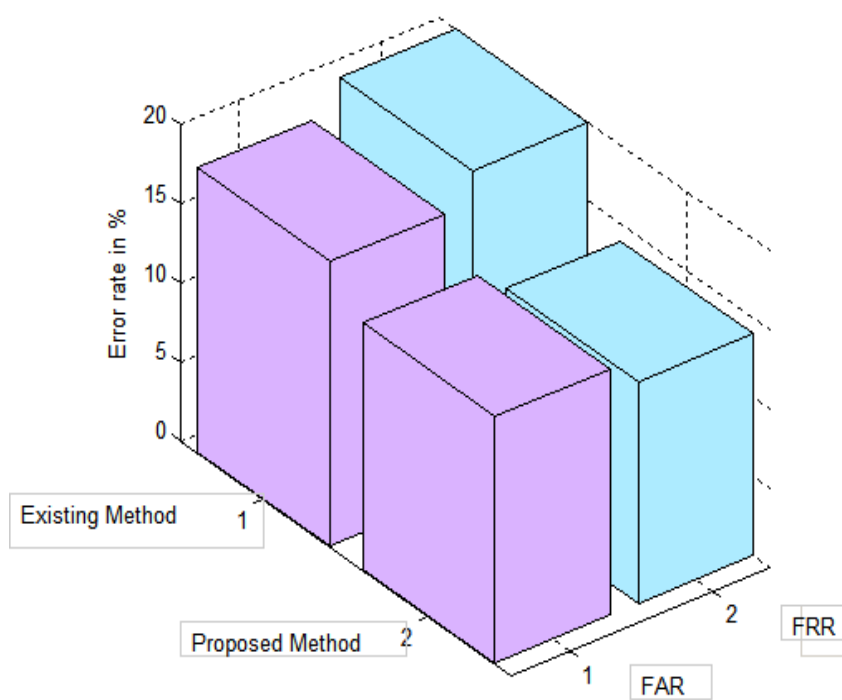

Fig 19: FAR and FRR analysis 


\section{CONCLUSION}

The iris biometrics gives out the unique result for individual human identity. The visible part of iris region was affected by the eyelids and eye lashes. The proposed method effectively eliminates the iris hazards. The morphological operator exactly finds pupil region. The Proposed algorithm exactly finds iris regions. The exact iris region was useful for finding the unique key feature. The key features were extracted independently from the left and right iris. The image matching technique performs the two pass comparison, which reduces the FRR to $14 \%$ and FAR to $15.6 \%$. This error rates were comparatively reduced value and which in turn increase the recognition rate.

\section{ACKNOWLEDGEMENT}

We thank the Chinese Academy of Science, Institute of Automation (CASIA), for providing the iris data base for testing out the proposed algorithm. We also thank our institution for conducting our research work.

\section{REFERENCES}

[1] Pierre E. Abi-Char, Bachar El-Hassan, Abdallah Mhamed. 2011. An Enhanced Authenticated Key Agreement Protocol with a Neural Network-based Model for Joining-Phase in Mobile Environments, International Journal of Engineering and Industries, Vol.2, No. 2.

[2] Thiyaneswaran B, Kandiban R, Jayakumar K.S. 2012. Localization of iris region using MLRP algorithm intended for biometric applications, European journal of scientific research(EJSR), Vol. 74, Issue 1, pp.126-133

[3] Debnath Bhattacharyya, Rahul Ranjan, Farkhod Alisherov A. and Minkyu Choi. 2009. Biometric Authentication, A Review, International Journal of uand e- Service, Science and Technology Vol. 2, No. 3.

[4] Chowhan.S.S., Shinde.G.N, COCSIT, Latur. 2008. Iris biometric recognition application in security management, Image and Signal Processing, CISP '08, pp.661 - 665 .

[5] L. Masek. 2003. Recognition of Human Iris Patterns for Biometric Identification M. Thesis, The University of Western Australia.Vol.3.

[6] Bhawna Chohan, Shailija Shukla. 2010. Analysis of statistical feature extraction for iris recognition system using Laplacian if Gaussian filter, International journal of applied engineering research, Vol.1.

[7] Anguraj K, Kandiban R, Jayakumar K S. 2012. Facial paralysis diseases level detection using CEM algorithm for clinical applications, European journal of scientific research, Vol77, Issue.4, PP 543-548.

[8] Jong Gook Ko, Youn Gil, Kvo Chung. 2007. A Noval and efficient feature extraction method for iris recognition, ETRI Journal, Vol.29, PP 399-401.

[9] Birgale, L.V., Kokare, M. 2009. Iris recognition using discrete wavelet transform, Digital Image Processing, International Conference, pp.147 - 151.

[10] Zhongliang Luo, Tusheng Lin., 2008. Detection of Noniris Region in the Iris Recognition, Computer Science and Computational Technology, ISCSCT '08, pp.45-48.

[11] Wibowo, E.P., Maulana, W.S. 2009. Real-Time Iris Recognition System Using a Proposed Method, International Conference on Signal Processing Systems, pp. $98-102$.

[12] J. Sauvola, M. Pietikäinen. 2000. Adaptive document image binarization. Pattern Recognition 33, pp. 225-236.

[13] Asano, M., Takano, H., Nakamura, K. 2010. Iris detection method using particle filter and edge directional features, World Automation Congress (WAC), pp.1-6.

[14] Horapong. K., Sreecholpech. J., Thainimit. S., Areekul. V. 2005. An Iris Verification Using Edge Detection, Information, Communications and Signal Processing, pp. $1434-1438$.

[15] Topi Maenpaa. 2005. An Iterative Algorithm for Fast Iris Detection. IWBRS. Beijing, China. pp.127-134.

[16] Srinivasa kumar, G.Ramaswamy, D.Ravikiran, P.sirisha rani. 2009. A novel approach for an accurate human Identification through iris recognition using bit plane slicing and normalization, Journal of theoretical and applied information technology,pp.531-537.

[17] Arti Dhiman, Ashok Kumar, Manoj Arora. 2011 Design of a Real Time System for Acquiring and Analyzing Iris Images in MATLAB, IJECT, Vol. 2, Issue 3, PP.106-110.

[18] Christopher Boyce, Arun Ross, Matthew Monaco, Lawrence Hornak and Xin Li. 2006. Multispectral Iris Analysis: A Preliminary Study, International Workshop On Computer Biometrics, pp 211-216.

[19] Gite h.r., Mahender C.N. 2011. Iris Code Generation and Recognition, International Journal of Machine Intelligence, Volume 3, Issue 3, pp-103-107. 